\title{
Déficits en la acción socioeducativa dirigida a adolescentes vulnerables latinoamericanos residentes en España
}

\author{
Deibe Fernández-Simo, Ph.D. \\ Universidad de Vigo, España*
}

Xosé Manuel Cid-Fernández, Ph.D.

Universidad de Vigo, España**

María Victoria Carrera-Fernández, Ph.D.

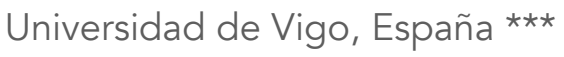

jesfernandez@uvigo.es

\section{Resumen (analítico)}

El Sistema de Protección a la Infancia y a la Adolescencia español utiliza los mismos mecanismos con la población tutelada nacional que con la extranjera de origen latinoamericano. Con esta investigación se pretende conocer las dificultades específicas de la juventud procedente de América Latina, en edad de emancipación y que tiene expediente de protección, así como identificar estrategias eficaces para la actuación socioeducativa. Mediante metodología cualitativa, en el marco de la teoría fundamentada, se realizan con juventud tutelada seis seguimientos longitudinales y cuatro relatos de vida. Se concluye con dos grupos de discusión con figuras profesionales especializadas. Los resultados muestran déficits en el acompañamiento socioeducativo, especialmente producto del choque entre las metas del proyecto migratorio y la rigidez del sistema. Se constata la ausencia de redes sociales de apoyo para la emancipación.

\section{Palabras clave}

Migración latinoamericana, emancipación, menores no acompañados, bienestar de la infancia, programa social, cambio social.

\section{Thesauro}

Tesauro de Ciencias Sociales de la Unesco.

\section{Para citar este artículo}

Fernández-Simo, D., Cid-Fernández, X. M., \& Carrera-Fernández, M. V. (2020). Déficits en la acción socioeducativa dirigida a adolescentes vulnerables latinoamericanos residentes en España. Revista Latinoamericana de Ciencias Sociales, Niñez y Juventud, 18(2), 1-24. http://dx.doi.org/ $10.11600 / 1692715 x .18202$

\section{Historial}

Recibido: 14.06 .2019

Aceptado: 01.08.2019

Publicado: 16.04.2020

\section{Información artículo}

Este artículo se basa en una investigación longitudinal más amplia iniciada en septiembre del 2010 y que continúa activa, referente a la «Intervención socioeducativa con adolescentes en protección y el impacto en sus itinerarios vitales». Los autores realizan el trabajo con el apoyo del Departamento de Análisis e Intervención Psicosocioeducativa de la Universidad de Vigo. Número de Identificación: Q8650002B. Área: Ciencias de la educación. Subárea: Educación especial general. 


\section{Deficits in educational action with vulnerable adolescents from Latin America living in Spain}

Abstract (analytical)

The Protection System for Children and Adolescents in Spain has the same mechanisms for the national population as it does for the foreign population of children and Young people who are originally from Latin American. This research aimed to determine the specific emancipation difficulties of youths from Latin America with a Child Protective Service record, as well as to identify effective strategies for socio-educational actions. Using a qualitative methodology within the grounded theory framework, six longitudinal monitoring studies and four life story narrations are conducted with protected young people. The study concluded with two discussion groups with specialized professional figures. The results reveal deficits in educational accompaniment, especially as a result of the collision between migratory project goals and the rigidity of the education system. The absence of social support networks to contribute to their emancipation is confirmed.

Keywords

Latin American migration, emancipation, unaccompanied minors, child welfare, social program, social change.

\section{Déficits na ação socio-educacional com adolescência vulnerável da América Latina e residente na Espanha}

\section{Resumo (analítico)}

O Sistema de Proteção da Infância e da Adolescência Espanhola, atua com os mesmos mecanismos com a população tutelada nacional estrangeira e com a de origem latino-americana. A investigação pretende conhecer as dificuldades específicas da juventude, com expedientes de proteção com as nacionalidades da américa latina, durante a emancipação, assim como identificar estratégias eficazes para a atuação socioeducativa, mediante uma metodologia qualitativa. O marco da teoria fundamentada, realiza-se com juventude tutelada de seis seguimentos longitudinais e de quatro relatos de vida. Conclui com grupos de discussão com figuras profissionais especializadas. Os resultados mostram déficits em acompanhamento socioeducativo, especialmente produto de choque entre as metas de projeto migratório e da rigidez do sistema. Constata-se a ausência de redes sociais de apoio para a emancipação.

\section{Palavras-chave}

Migração latino americana, emancipaçao, menores extrangeiros desacompanhados, bem-estar infantil, programa social, mudança social.

Información autores

[*] Graduado en Educación Social. Doctor en Ciencias de la Educación por la Universidad de Vigo. Índice H5: 2. Correo electrónico: jesfernandez@uvigo.es iD 0000-0001-6202-4452.

[**] Licenciado en Pedagogía. Doctor en Pedagogía por la Universidad de Santiago de Compostela. Índice H5: 4. Correo electrónico: xcid@uvigo.es (iD) 0000-0002-7470-1737.

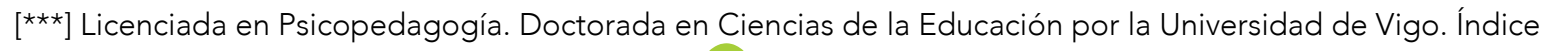
H5: 18. Correo electrónico: mavicarrera@uvigo.es (iD) 0000-0003-2158-3084. 


\section{Introducción}

$\mathrm{C}_{\text {asumir el cuidado de la juventud y la adolescencia - tanto nacional como de }}$ origen extranjero- en situación de vulnerabilidad. Investigaciones recientes destacan la importancia de que los y las jóvenes no nacionales en dificultad social que se encuentran en España deben ser objeto de políticas sociales que cuenten con estrategias de respuesta a sus necesidades específicas (Ruiz et al., 2019); ello dado que la presencia de juventud extranjera en los sistemas de protección de Europa se ha consolidado y ha pasado a ser un fenómeno que persistirá en el tiempo (Gimeno, 2018).

Los condicionantes diferenciadores del colectivo son resultado de las características propias de cada itinerario vital, determinado por las vivencias experimentadas durante el proceso de migración. Estas se podrían denominar heridas (Martín et al., 2016) producto de las duras experiencias presentes en todo desplazamiento hacia lugares desconocidos. Las vivencias durante el trayecto y el proceso de adaptación a la sociedad de destino son factores relevantes para valorar cada caso (Hopkins \& Hill, 2010). Por ende, la realidad vivida por cada joven migrante es crucial para diagnosticar sus necesidades socioeducativas, las cuales serán la base para construir la estrategia de acompañamiento profesional.

La trayectoria migratoria del o de la joven debería condicionar la respuesta específica que el Sistema de Protección construye en cada caso. Según Monteros (2014), el itinerario migratorio y la experiencia de pertenencia a los espacios de llegada son factores determinantes para atender a los y las jóvenes migrantes. Los condicionantes expuestos implican que el papel referencial de los equipos educativos es incluso más relevante que el cumplido con la juventud española, y la forma como se sitúan las figuras profesionales frente a ellos y ellas es un elemento facilitador de la construcción de la identidad personal en el marco del contexto de llegada (López, 2018). La ausencia de redes naturales (que en la mayoría de los casos quedaron en el país de origen) determina la importancia de facilitar un soporte de confianza que, durante un proceso de resiliencia, permita marchar hacia la autonomía. 
Iniciamos el presente trabajo con la intención de avanzar en el conocimiento de la situación que la juventud nacida en América Latina vive en el Sistema de Protección de España. En el Estado español el bienestar social es competencia y responsabilidad de las comunidades autónomas. La investigación que presentamos se contextualiza en Galicia. El Gobierno gallego dispone de una diversa red de recursos especializados con los que pretende atender la infancia y la adolescencia en riesgo o desamparo. Entre los años 2012 y 2015, las estadísticas de la Xunta de Galicia (2016) muestran una media de 40.75 expedientes anuales de menores con nacionalidades latinoamericanas. En las estadísticas oficiales observamos una diferencia significativa en la medida de protección adoptada según la procedencia del menor. El $43.7 \%$ de la adolescencia residente en centros es extranjera, mientras que solo el 9.6\% de la infancia acogida por una familia tiene alguna nacionalidad diferente de la española (Ministerio de Sanidad, Consumo y Bienestar Social, 2018). En el caso de Galicia, los adolescentes no españoles que residen con familias de acogida suponen el $21.3 \%$ del total de beneficiados con esta medida.

La representatividad de la juventud de origen latinoamericano se incrementa si se compara con el total de la juventud migrante acompañada por el Sistema de Protección durante la emancipación. Según datos obtenidos de las memorias de la entidad gestora del Programa de Transición a la Vida Adulta (Igaxes3, 2016), en 2011 los menores latinoamericanos representaban el $21 \%$ del total de extranjeros, mientras que para el 2015 habían ascendido al $48.05 \%$. Teniendo en cuenta que los recursos de emancipación son pieza clave y el último eslabón del entramado de protección en el proceso de empoderamiento, la representatividad de la adolescencia latinoamericana en el sistema gallego está en incremento. La atención a este colectivo es un reto para la administración responsable de la política social en Galicia.

Las condiciones en las que se inicia la vida independiente son el principal elemento de valoración de la calidad de la actuación socioeducativa, ejecutada al amparo de las medidas de protección. Así, nos planteamos los objetivos que pasamos a enumerar: 1) conocer las dificultades específicas de la juventud de origen latinoamericano con expediente de protección durante la planificación del proceso de emancipación apoyado con los recursos de protección; 2) identificar las estrategias de los equipos profesionales que resultaron más eficaces en la colaboración prestada a la consecución de las metas personales; 3) identificar aspectos que puedan mejorarse en el acompañamiento socioeducativo brindado a la juventud de origen latinoamericano. 


\section{Método}

Mediante el presente trabajo de investigación, de naturaleza cualitativa, se pretende conocer aspectos propios de un contexto de intervención socioeducativa caracterizado por la cercanía de las relaciones personales. La acción analizada tiene lugar en el sur de Galicia, tanto en la ciudad de Ourense como en las villas dispersas por todo el territorio. Se analiza el acompañamiento que se les presta a jóvenes que cuentan con expediente administrativo de protección abierto por la Consellería de Política Social del Gobierno gallego. Las vivencias previas a la actuación protectora tienen como consecuencia la necesidad, en la persona, de reconstruir los espacios de seguridad personal (Puig \& Rubio, 2015) imprescindibles para asumir las metas del itinerario de inclusión social (Schütz et al., 2015). La obtención de información real y significativa debe apoyarse en la voz de los actores implicados (Denzin \& Lincoln, 2008), en este caso, la juventud latinoamericana y los profesionales del Sistema de Protección a la Infancia. Utilizamos técnicas de investigación que posibilitan la perspectiva dialógica, ya que la información obtenida tiene que ser creíble para los protagonistas, primeros conocedoros de la situación que se investiga (Miles \& Huberman, 2007). Las metodologías participativas son especialmente interesantes para el fomento del empoderamiento de los participantes (Puigvert, 2014), así como para la transformación de su propia realidad (Tójar, 2006).

Organizamos el trabajo en dos fases: en la primera se realizaron los seguimientos longitudinales y se obtuvieron los relatos de vida de los jóvenes que contaban con expediente de tutela en el Sistema de Protección a la Infancia y a la Adolescencia de Galicia. En la segunda se organizaron dos grupos de discusión con profesionales de la educación social que trabajan acompañando a jóvenes con dificultad social.

La primera fase se ejecutó entre octubre del 2015 y julio del 2016 mediante el acompañamiento socioeducativo a jóvenes que habían dejado de vivir en centros residenciales de protección de Galicia y a partir de la construcción de sus relatos de vida. La emancipación es un proceso marcado por la temporalidad, lo que hace aconsejable un seguimiento, para el que la investigación cualitativa longitudinal es especialmente indicada (Holland et al., 2006). El acompañamiento, en este caso a tres jóvenes, se realizó durante los seis primeros meses de su vida independiente. Ellos mantenían abierto un expediente administrativo con medida de apoyo familiar. La observación la efectuó la propia figura profesional durante los primeros momentos de la transición a la vida adulta, quien procuraba minimizar el impacto que podría producir la presencia del investigador (Ruiz, 
2012). Esta técnica nos permitió registrar fenómenos de interés para los objetivos del trabajo, que se contrastaron con los resultados de los relatos de vida. En este caso, los jóvenes emancipados reflexionaron sobre su experiencia vital con los recursos de protección, así como sobre los aspectos relevantes durante su proceso de transición a la vida adulta. Se inició la construcción de los relatos de vida con seis participantes. El criterio de elección de la muestra es que llevaron al menos seis meses fuera del Sistema de Protección. Se completó la obtención de la información en cuatro casos (tabla 1).

\section{Tabla 1}

Muestra de juventud de origen latinoamericano con expediente de tutela en Galicia

\begin{tabular}{|c|c|c|c|c|}
\hline Técnica & Código & Sexo & País de origen & Edad \\
\hline \multirow{3}{*}{$\begin{array}{l}\text { Seguimiento } \\
\text { Longitudinal }\end{array}$} & SL1 & Masculino & Colombia & 20 \\
\hline & SL2 & Femenino & Colombia & 19 \\
\hline & SL3 & Femenino & República dominicana & 18 \\
\hline \multirow{4}{*}{$\begin{array}{c}\text { Relatos de } \\
\text { vida }\end{array}$} & RV1 & Masculino & Colombia & 21 \\
\hline & RV2 & Femenino & Colombia & 23 \\
\hline & RV3 & Femenino & Brasil & 19 \\
\hline & RV4 & Masculino & Paraguay & 19 \\
\hline
\end{tabular}

El proceso consistió en la realización de tres entrevistas en profundidad orientadas a conocer cómo los jóvenes participantes entendían la realidad estudiada (Steinar, 2011). La primera buscó un acercamiento a los temas de interés para la investigación. En la segunda se profundizó en la cuestión. En la última entrevista se pusieron a disposición de los y las informantes los resultados obtenidos en las anteriores, con la intención de que ratificaran o rectificaran lo que consideraran oportuno. Todos los participantes eran mayores de edad. Firmaron un consentimiento informado en el que constaba el propósito de la investigación. Es destacable la motivación con la que los jóvenes accedieron a participar en el presente trabajo, argumentando la necesidad de mejorar los recursos de atención.

Los resultados de la fase anterior fueron puestos a disposición de las figuras profesionales en dos grupos de discusión, celebrados en septiembre y octubre del 2016 (tabla 2). Esta técnica permite conocer, de un modo intensivo, las percepciones sobre el problema objeto de estudio. Los grupos están abiertos a todo tipo de opiniones, independientemente de su naturaleza (Báez, 2007), para lo cual se promueve un ambiente en el que las opiniones surjan de forma natural. Elegimos una propuesta metodológica secuenciada, 
que posibilita una triangulación diferida que, mediante la utilización de diferentes técnicas, facilita la configuración de categorías que representan fenómenos significativos para las personas que participan (Strauss \& Corbin, 2002). Los grupos de discusión estuvieron compuestos por educadores y educadoras sociales que prestan acompañamiento socioeducativo en el tema de recursos de protección. El proceder metodológico, de naturaleza dinámica y flexible, resulta especialmente apropiado para contextos de naturaleza singular, cuya transferibilidad es posible en realidades con condiciones y características similares (San Martín, 2014). Avanzamos hacia una teorización sustantiva, propia de la teoría fundamentada.

\section{Tabla 2}

Muestra de grupos de discusión con figuras profesionales

\begin{tabular}{|c|c|c|}
\hline & Código & $\begin{array}{l}\text { Años de experiencia } \\
\text { laboral específica }\end{array}$ \\
\hline \multirow{4}{*}{ Grupo de discusión 1} & GD1P1P & 8 \\
\hline & GD1P2P & 17 \\
\hline & GD1P3P & 9 \\
\hline & GD1P4P & 7 \\
\hline \multirow{5}{*}{ Grupo de discusión 2} & GD2P1P & 1 \\
\hline & GD2P2P & 2 \\
\hline & GD2P3P & 14 \\
\hline & GD2P4P & 5 \\
\hline & GD2P5P & 6 \\
\hline
\end{tabular}

La credibilidad de la información, apoyada en la triangulación de técnicas, fue reforzada con el diseño secuenciado del proceso. Los resultados obtenidos con la juventud en dificultad social fueron contrastados por figuras profesionales conocedoras de la cuestión. La confirmabilidad fue garantizada por la supervisión externa de todo el proceso, ejecutada por dos profesionales con reconocida experiencia en el trabajo con adolescentes (Martínez, 2006). La supervisión de expertos contribuyó a dar coherencia a los resultados (Sandin, 2003). El proceso de investigación fue diseñado para garantizar la representatividad de las categorías resultantes. 


\section{Resultados}

A su llegada, la juventud de origen latinoamericano se encuentra con una barrera que supondrá una dificultad decisiva en su proceso de emancipación: el acceso a un sistema educativo rígido que no tiene en cuenta los factores sociales y que trata a todo el alumnado con un falso igualitarismo, olvidando que la realidad personal de cada estudiante es diferente. Esta situación se traduce en la vivencia de procesos de exclusión académica: «me metieron en un curso en el que no me enteraba de nada: hablaban de cosas que yo nunca había estudiado», manifiesta una joven $\left(R V_{2}\right)$. «Si a las dificultades existentes de integración en la escuela le sumas que metes al chico en un curso para el que no tiene nivel académico suficiente, ya tienes la fórmula perfecta para que se produzca el fracaso escolar», dice un educador $\left(\mathrm{GD}_{1} \mathrm{P}_{2} \mathrm{P}\right)$. Se constata la ausencia de figuras de referencia que promuevan la inclusión en el ámbito educativo; el propio entorno relacional presenta dinámicas que chocan con las demandas escolares (SL1, SL2).

La escuela no solo no es sensible a las demandas sociales, sino que es implacable en la exclusión del alumnado que no se adapta a sus pretensiones: «en el colegio lo que les importa es el contenido y el comportamiento; el resto les da igual», manifiesta una educadora $\left(\mathrm{GD}_{2} \mathrm{P}_{5} \mathrm{P}\right)$. «Hay muchos estigmas presentes en los profesores, que mayoritariamente permanecen ajenos a la realidad social», comenta una profesional $\left(\mathrm{GDIP}_{4} \mathrm{P}\right)$. En la actuación de la escuela, la ausencia del factor social es sobradamente conocida. Esta situación perjudica sobre todo al alumnado que presenta necesidades sociales; por lo tanto, incide negativamente en la juventud de origen latinoamericano.

La medida administrativa de tutela conlleva la posibilidad de acceso a una autorización de residencia e, incluso, de trabajo; esta última en caso de que el equipo técnico del menor considere positivo que, para su proceso de integración, realice actividad laboral. La contradicción de la acción protectora surge cuando se cumple la mayoría de edad, momento en el que, para la renovación de la autorización, es preciso contar con un contrato de trabajo de un año de duración. Esta exigencia administrativa constituye un déficit específico en la atención a la juventud migrante. Así, la construcción burocrática de la entidad pública resulta un factor limitador determinante en la posibilidad de inserción social (tabla 3). 
Tabla 3

Factores obstaculizadores del proceso de emancipación de jóvenes de origen latinoamericano que reciben protección

\begin{tabular}{|c|c|c|c|c|c|c|}
\hline \multirow[b]{2}{*}{ CE } & \multirow{2}{*}{\multicolumn{3}{|c|}{ Exclusión del sistema educativo }} & \multicolumn{3}{|c|}{ Frecuencia } \\
\hline & & & & $\begin{array}{l}\text { Relatos } \\
\text { de vida }\end{array}$ & $\begin{array}{l}\text { Seguimientos } \\
\text { longitudinales }\end{array}$ & $\begin{array}{l}\text { Grupos de } \\
\text { discusión }\end{array}$ \\
\hline CE1 & \multicolumn{3}{|c|}{$\begin{array}{l}\text { Acceso al sistema educativo en curso académico según la edad cronológica } \\
\text { del alumno, pero con diferencias curriculares significativas }\end{array}$} & $\begin{array}{l}\text { RV1 } \\
\text { RV2 } \\
\text { RV3 } \\
\text { RV4 }\end{array}$ & $\begin{array}{l}\text { SL1 } \\
\text { SL2 } \\
\text { SL3 }\end{array}$ & $\begin{array}{l}\text { GD1P1P } \\
\text { GD1P2P } \\
\text { GD1P3P } \\
\text { GD2P1P } \\
\text { GD2P2P }\end{array}$ \\
\hline CE2 & \multicolumn{3}{|c|}{ Dificultades específicas de integración en el centro escolar } & $\begin{array}{l}\mathrm{RV} 1 \\
\mathrm{RV} 2 \\
\mathrm{RV} 3 \\
\mathrm{RV} 4\end{array}$ & $\begin{array}{l}\text { SL1 } \\
\text { SL2 } \\
\text { SL3 }\end{array}$ & $\begin{array}{l}\text { GD1P1P } \\
\text { GD1P2P } \\
\text { GD1P4P } \\
\text { GD2P1P } \\
\text { GD2P5P }\end{array}$ \\
\hline CE3 & \multicolumn{3}{|c|}{ Ausencia de referentes personales favorecedores de la inclusión académica } & $\begin{array}{l}\mathrm{RV} 2 \\
\mathrm{RV} 3 \\
\mathrm{RV} 4\end{array}$ & $\begin{array}{l}\mathrm{SL2} \\
\mathrm{SL3}\end{array}$ & $\begin{array}{l}\text { GD1P1P } \\
\text { GD1P2P } \\
\text { GD1P3P } \\
\text { GD1P4P } \\
\text { GD2P1P } \\
\text { GD2P2P } \\
\text { GD2P5P }\end{array}$ \\
\hline \multirow[b]{2}{*}{ CA } & \multirow{2}{*}{\multicolumn{3}{|c|}{ Obstáculos en el proceso de inserción laboral }} & \multicolumn{3}{|c|}{ Frecuencia } \\
\hline & & & & $\begin{array}{l}\text { Relatos } \\
\text { de vida }\end{array}$ & $\begin{array}{l}\text { Seguimientos } \\
\text { longitudinales }\end{array}$ & $\begin{array}{l}\text { Grupos de } \\
\text { Discusión }\end{array}$ \\
\hline CA1 & \multicolumn{3}{|c|}{$\begin{array}{l}\text { Exigencias legales para la autorización de trabajo que dificulta la } \\
\text { consecución de un empleo }\end{array}$} & $\begin{array}{l}\text { RV3 } \\
\text { RV4 }\end{array}$ & $\begin{array}{l}\mathrm{SL2} \\
\mathrm{SL3}\end{array}$ & $\begin{array}{l}\text { GD1P1P } \\
\text { GD1P2P } \\
\text { GD1P3P } \\
\text { GD2P1P } \\
\text { GD2P2P } \\
\text { GD2P4P }\end{array}$ \\
\hline CA2 & \multicolumn{3}{|c|}{ Actitud personal en conflicto con las demandas del mercado laboral } & $\begin{array}{l}\text { RV1 } \\
\text { RV3 } \\
\text { RV4 }\end{array}$ & $\begin{array}{l}\text { SL2 } \\
\text { SL3 }\end{array}$ & $\begin{array}{l}\text { GD1P1P } \\
\text { GD1P4P } \\
\text { GD2P2P } \\
\text { GD2P4P } \\
\text { GD2P5P }\end{array}$ \\
\hline CA3 & \multicolumn{3}{|c|}{ Dificultades de acceso a la formación para el empleo } & $\begin{array}{l}\mathrm{RV} 1 \\
\mathrm{RV} 2 \\
\mathrm{RV} 3 \\
\mathrm{RV} 4\end{array}$ & $\begin{array}{l}\text { SL1 } \\
\text { SL2 } \\
\text { SL3 }\end{array}$ & $\begin{array}{l}\text { GD1P1P } \\
\text { GD1P2P } \\
\text { GD1P3P } \\
\text { GD2P2P } \\
\text { GD2P3P } \\
\text { GD2P5P }\end{array}$ \\
\hline \multirow[b]{2}{*}{$\mathrm{CB}$} & \multirow{2}{*}{\multicolumn{3}{|c|}{$\begin{array}{l}\text { Insuficiencia de ingresos económicos necesarios para hacer frente a los } \\
\text { gastos propios de la vida diaria }\end{array}$}} & \multicolumn{3}{|c|}{ Frecuencia } \\
\hline & & & & $\begin{array}{l}\text { Relatos } \\
\text { de vida }\end{array}$ & $\begin{array}{l}\text { Seguimientos } \\
\text { longitudinales }\end{array}$ & $\begin{array}{l}\text { Grupos de } \\
\text { Discusión }\end{array}$ \\
\hline CB1 & \multicolumn{3}{|c|}{ Empleo a jornada parcial } & $\begin{array}{l}\text { RV1 } \\
\text { RV3 }\end{array}$ & $\begin{array}{l}\text { SL1 } \\
\text { SL2 } \\
\text { SL3 }\end{array}$ & $\begin{array}{l}\text { GD1P1P } \\
\text { GD1P2P } \\
\text { GD1P3P } \\
\text { GD2P1P } \\
\text { GD2P2P } \\
\text { GD2P4P }\end{array}$ \\
\hline CB2 & \multicolumn{3}{|c|}{ Actividad laboral esporádica } & $\begin{array}{l}\mathrm{RV} 1 \\
\mathrm{RV} 2 \\
\mathrm{RV} 3\end{array}$ & $\begin{array}{l}\text { SL1 } \\
\text { SL2 }\end{array}$ & $\begin{array}{l}\text { GD1P2P } \\
\text { GD1P4P } \\
\text { GD2P1P } \\
\text { GD2P2P } \\
\text { GD2P3P } \\
\text { GD2P4P }\end{array}$ \\
\hline \multirow[b]{2}{*}{ CB3 } & \multirow{2}{*}{$\begin{array}{l}\text { Ausencia de colchón } \\
\text { social }\end{array}$} & CB3.1 & No presencia de referentes familiares & $\begin{array}{l}\mathrm{RV} 2 \\
\mathrm{RV} 3 \\
\mathrm{RV} 4\end{array}$ & $\begin{array}{l}\mathrm{SL} 1 \\
\mathrm{SL2}\end{array}$ & $\begin{array}{l}\text { GD1P1P } \\
\text { GD1P3P } \\
\text { GD2P3P }\end{array}$ \\
\hline & & CB3.2 & $\begin{array}{l}\text { Ausencia de red asociativa de apoyo del } \\
\text { país de origen }\end{array}$ & $\begin{array}{l}\mathrm{RV} 1 \\
\mathrm{RV} 2 \\
\mathrm{RV} 3 \\
\mathrm{RV} 4\end{array}$ & $\begin{array}{l}\text { SL1 } \\
\text { SL2 } \\
\text { SL3 }\end{array}$ & $\begin{array}{l}\text { GD1P2P } \\
\text { GD1P4P } \\
\text { GD2P1P } \\
\text { GD2P2P }\end{array}$ \\
\hline
\end{tabular}


Conscientes de la situación del mercado laboral, nos asomamos a un reto de elevada complejidad, tal como se demuestra en los relatos de vida y los seguimientos longitudinales $\left(\mathrm{RV}_{3}, \mathrm{RV}_{4}, \mathrm{SL}_{2}, \mathrm{SL}_{3}\right)$ : «la renovación de la autorización para trabajar implica mucho más que el contrato de un año, ya que la empresa contratante tiene que cumplir unos requisitos económicos, que muchas no cumplen», manifiesta una educadora (GD2 $\mathrm{P} 1 \mathrm{P})$. El estrés propio de la situación de desamparo se incrementa con la preocupación por la renovación de la autorización de trabajo.

Las dificultades específicas para acceder a la formación para el empleo son resultado de dos elementos comentados: las consecuencias de la exclusión escolar y las dificultades de regularización. La tardanza de los trámites burocráticos para la obtención de las autorizaciones incide negativamente en los tiempos de acceso a las acciones formativas para el empleo, tal y como se pudo comprobar en todos los relatos de vida y seguimientos realizados.

Otra dificultad que tiene mayor incidencia en las personas jóvenes de origen latinoamericano es la normativa aplicada a los certificados de profesionalidad, que supone exigencias de cualificación para las formaciones de nivel II, lo que a gran parte de esta población le impide contar con esta oportunidad, sobre todo si se tiene en cuenta que la mayor oferta formativa se corresponde con cursos de este nivel: «aprender a realizar un trabajo es fundamental para acceder a un empleo, pero cursos a los que puedas entrar sin la ESO son muy pocos», comenta una profesional $\left(\mathrm{GD}_{2} \mathrm{P} 3 \mathrm{P}\right)$. Otra educadora nos aproxima a la cuestión temporal, en la que se observa una contradicción entre las urgencias derivadas de los tiempos de intervención durante la emancipación y la dilatación de los trámites administrativos de las autorizaciones: «se pierde mucho tiempo en cuestiones burocráticas, y tenemos poco tiempo, ya que hay que prepararse para la vida independiente en poco más de un año» $\left(\mathrm{GD}_{2} \mathrm{P}_{4} \mathrm{P}\right)$.

Un aspecto fundamental que debe tenerse en cuenta es el de las tensiones entre las motivaciones del itinerario migratorio y las pretensiones institucionales. El régimen normativo de los recursos entra frecuentemente en conflicto con la organización vital de las y los jóvenes: «la norma se aplica, en muchas ocasiones, sin que el chico entienda el significado educativo de la incidencia», comenta una profesional $\left(\mathrm{GD}_{2} \mathrm{P}_{5} \mathrm{P}\right)$. «Deberíamos destacar más durante la relación con ellos las posibilidades que tiene el niño, sin centrarnos tanto en las cosas negativas», manifiesta una educadora $\left(\mathrm{GD}_{2} \mathrm{P}_{2} \mathrm{P}\right)$.

El trabajo en red presenta déficits, tanto en la acción coordinada de los propios recursos del sistema como en la colaboración con organizaciones e instituciones externas. En todos los seguimientos longitudinales se detectaron aspectos en los que la coordina- 
ción fue deficitaria. La totalidad de las figuras profesionales que participaron en los grupos de discusión destacan la conveniencia de mejorar la actuación coordinada: «desconocemos el trabajo que se realiza en otros centros por los que pasaron chicos con los que estamos trabajando», afirma una educadora $\left(\mathrm{GD}_{2} \mathrm{P}_{3} \mathrm{P}\right)$. «Parece como si una vez que se va a otro lugar, ya es asunto de otro equipo, y no puede ser así», dice una profesional $\left(\mathrm{GD}_{1} \mathrm{P}_{4} \mathrm{P}\right)$. «La información no fluye entre centros, no sabemos qué se hizo en los que estuvo antes el chico», comenta una educadora $\left(\mathrm{GD}_{2} \mathrm{P}_{4} \mathrm{P}\right)$.

La juventud tutelada originaria de América Latina padece especialmente las consecuencias de la falta de adecuación de los procesos administrativos de protección a la realidad actual de la emancipación. La reversibilidad de los itinerarios de transición a la adultez no encuentra una respuesta adecuada en la acción educativa. En el caso de la juventud migrante latina, la situación es incluso más preocupante, por no contar con apoyos referenciales efectivos: «trabajé con chicas de Colombia y República Dominicana, y no hay una persona adulta, ni una asociación, a quien recurrir para que sean apoyo», dice una educadora $\left(\mathrm{GD}_{2} \mathrm{P}_{4} \mathrm{P}\right)$. «Para los africanos contamos con una red asociativa que apoya el trabajo, pero para los de Colombia no hay una sola asociación, y eso que la población es muy amplia», comenta un profesional (GD1P2P). Otra educadora lamenta que las propuestas del sistema para dar cobertura de referentes a la juventud no cuentan con voluntarios nacionales de los países de referencia: «los programas de referenciado social no cuentan con la participación de emigrantes latinoamericanos, que voluntariamente colaboren como adultos de apoyo», afirma una educadora (GD1P1P).

Los déficits constatados determinan la planificación de las estrategias más eficaces de los equipos educativos, ya que condicionan la evolución del acompañamiento, y es imperativa su contemplación por las figuras profesionales durante el diseño de la actuación socioeducativa. Anteriormente señalábamos el proceso de exclusión académica como la primera de las barreras institucionales detectadas. La percepción de fracaso en los temas escolares tiene consecuencias en la seguridad personal e influye en la disposición para asumir las metas propias de la transición a la adultez. La propia vivencia de la exclusión escolar tiene consecuencias negativas en la motivación para afrontar nuevos objetivos personales. La ausencia de referentes personales en los que observar las dinámicas propias de integración en la actividad laboral dificulta la interiorización natural de las habilidades necesarias para la inclusión en el mercado de trabajo. Se constata un conflicto entre la actitud de la juventud y las demandas del ámbito laboral (RV1, RV 3 , $\left.\mathrm{RV}_{4}, \mathrm{SL}_{2}, \mathrm{SL}_{3}\right)$ : «llegas al trabajo y estás acostumbrado a actuar como tú entiendes que 
debes de actuar, y tienes problema con los jefes», manifiesta un joven (RV1E2). «La actitud es un aspecto crucial en el mantenimiento del empleo, y resulta muy complicado el proceso de acercamiento a las exigencias del puesto de trabajo», comenta una educadora ( GD1P1P). «Muchas veces en los centros insistimos en la conveniencia de estudiar, pero el joven quiere trabajar. Si se quiere buscar empleo, hay que actuar en esto, y listo», comenta una educadora $\left(\mathrm{GD}_{2} \mathrm{P}_{4} \mathrm{P}\right)$.

Las figuras profesionales configuran estrategias en las que se observa el clima de convivencia del recurso como un factor relevante en la eficacia del acompañamiento socioeducativo (tabla 4 ). La horizontalidad de las relaciones entre equipo educativo y los adolescentes favorece la implicación de estos últimos en su proyecto educativo: «es importante que sientan que son escuchados y que su PEI [Proyecto Educativo Individualizado] es suyo, que son ellos quienes lo montan», manifiesta una profesional ( $\left.\mathrm{GD}_{2} \mathrm{P}_{2} \mathrm{P}\right)$. Dinámicas dialógicas y participación son dimensiones que van de la mano y que están presentes en las argumentaciones analizadas. Una educadora afirma que «los chicos tienen que ver que te interesa su vida, que no son uno más» (GD1P3P). Una joven hace hincapié en esta cuestión afirmando que «sería bueno que fuésemos importantes para las educadoras, que no pareciese que vienen a trabajar y que no les importamos más» (RV2E3).

Otro factor determinante en la calidad del acompañamiento socioeducativo es la estabilidad de los equipos profesionales: «Las competencias profesionales de las educadoras son las que en mayor medida hacen posible el éxito de la intervención, sobre todo en lo que tiene que ver con la relación con los menores», dice un profesional ( $\left.\mathrm{GD}_{1} \mathrm{P}_{2} \mathrm{P}\right)$. Los equipos educativos configuran el nexo entre los adolescentes en protección y el sistema: «Los educadores mantenemos una relación intensa; las técnicas de la administración mantienen una relación distante, ya que están saturadas de casos y hay a chicos que ven una vez al año», comenta una educadora (GD1P1P).

La investigación revela que la estrategia del equipo educativo es de especial importancia para la juventud originaria de América Latina, ya que este colectivo carece de redes de apoyo social en Galicia: «Los menores suramericanos no tienen referentes positivos en la ciudad. Nosotros somos el nexo de unión entre su realidad cultural y la nuestra», comenta una profesional $\left(\mathrm{GD}_{2} \mathrm{P}_{1} \mathrm{P}\right)$. 
Tabla 4

Estrategias socioeducativas para el acompañamiento durante la transición a la vida adulta

\begin{tabular}{|c|c|c|c|c|c|c|}
\hline \multirow[b]{2}{*}{$\mathrm{CC}$} & \multirow{2}{*}{\multicolumn{3}{|c|}{$\begin{array}{c}\text { Implicación del joven en la consecución de las metas del itinerario de } \\
\text { emancipación }\end{array}$}} & \multicolumn{3}{|c|}{ Frecuencia } \\
\hline & & & & $\begin{array}{l}\text { Relatos de } \\
\text { vida }\end{array}$ & $\begin{array}{l}\text { Seguimientos } \\
\text { longitudinales }\end{array}$ & $\begin{array}{l}\text { Grupos de } \\
\text { discusión }\end{array}$ \\
\hline \multirow{3}{*}{ CC1 } & \multirow{3}{*}{ Clima de confianza } & $\mathrm{CC} 1.1$ & $\begin{array}{l}\text { Precisa horizontalidad en la relación } \\
\text { educativa }\end{array}$ & $\begin{array}{l}\mathrm{RV} 2 \\
\mathrm{RV} 3\end{array}$ & $\begin{array}{l}\text { SL1 } \\
\text { SL2 }\end{array}$ & $\begin{array}{l}\text { GD1P2P } \\
\text { GD1P3P } \\
\text { GD2P1P } \\
\text { GD2P2P } \\
\text { GD2P5P }\end{array}$ \\
\hline & & $\mathrm{CC} 1.2$ & $\begin{array}{l}\text { No visualización de la implicación de la } \\
\text { figura profesional }\end{array}$ & $\begin{array}{l}\mathrm{RV} 2 \\
\mathrm{RV} 3\end{array}$ & SL2 & $\begin{array}{l}\text { GD1P1P } \\
\text { GD1P2P } \\
\text { GD2P1P } \\
\text { GD2P3P }\end{array}$ \\
\hline & & $\mathrm{CC} 1.3$ & Presencia de participación aparente & $\begin{array}{l}\text { RV1 } \\
\text { RV2 } \\
\text { RV3 }\end{array}$ & $\begin{array}{l}\text { SL1 } \\
\text { SL2 } \\
\text { SL3 }\end{array}$ & $\begin{array}{l}\text { GD1P1P } \\
\text { GD1P2P } \\
\text { GD1P3P } \\
\text { GD2P1P } \\
\text { GD2P3P }\end{array}$ \\
\hline \multirow{4}{*}{$\mathrm{CC} 2$} & \multirow{3}{*}{ Adaptabilidad } & CC2.1 & No individualización & $\begin{array}{l}\text { RV1 } \\
\text { RV2 } \\
\text { RV3 }\end{array}$ & $\begin{array}{l}\text { SL1 } \\
\text { SL2 } \\
\text { SL3 }\end{array}$ & $\begin{array}{l}\text { GD1P1P } \\
\text { GD1P2P } \\
\text { GD2P2P } \\
\text { GD2P3P } \\
\text { GD2P4P } \\
\text { GD2P5P }\end{array}$ \\
\hline & & $\mathrm{CC} 2.2$ & $\begin{array}{l}\text { No contemplación de los tiempos del } \\
\text { proyecto migratorio }\end{array}$ & $\begin{array}{l}\text { RV1 } \\
\text { RV2 } \\
\text { RV3 }\end{array}$ & $\begin{array}{l}\text { SL2 } \\
\text { SL3 }\end{array}$ & $\begin{array}{l}\text { GD1P1P } \\
\text { GD1P2P } \\
\text { GD1P4P } \\
\text { GD2P1P } \\
\text { GD2P2P } \\
\text { GD2P3P }\end{array}$ \\
\hline & & $\mathrm{CC} 2.3$ & $\begin{array}{l}\text { Peso de las incidencias negativas } \\
\text { derivadas de las discrepancias entre } \\
\text { visión del joven y actuación institucional. } \\
\text { Déficit del peso de las fortalezas } \\
\text { personales. }\end{array}$ & $\begin{array}{l}\text { RV1 } \\
\text { RV2 } \\
\text { RV3 }\end{array}$ & $\begin{array}{l}\mathrm{SL} 2 \\
\mathrm{SL} 3\end{array}$ & $\begin{array}{l}\text { GD1P1P } \\
\text { GD1P3P } \\
\text { GD2P2P } \\
\text { GD2P3P } \\
\text { GD2P5P }\end{array}$ \\
\hline & & $\mathrm{CC} 2.4$ & Rigidez institucional & $\begin{array}{l}\text { RV2 } \\
\text { RV3 } \\
\text { RV4 }\end{array}$ & $\begin{array}{l}\mathrm{SL2} \\
\mathrm{SL3}\end{array}$ & $\begin{array}{l}\text { GD1P1P } \\
\text { GD1P2P } \\
\text { GD1P3P } \\
\text { GD1P4P } \\
\text { GD2P1P } \\
\text { GD2P2P } \\
\text { GD2P3P } \\
\text { GD2P4P } \\
\text { GD2P5P }\end{array}$ \\
\hline $\mathrm{CC} 3$ & \multicolumn{2}{|c|}{ Déficit de oferta de actividade } & pragmáticas & $\begin{array}{l}\text { RV1 } \\
\text { RV2 } \\
\text { RV4 }\end{array}$ & $\begin{array}{l}\text { SL1 } \\
\text { SL2 } \\
\text { SL3 }\end{array}$ & $\begin{array}{l}\text { GD1P1P } \\
\text { GD1P2P } \\
\text { GD1P3P } \\
\text { GD2P1P } \\
\text { GD2P2P } \\
\text { GD2P4P }\end{array}$ \\
\hline
\end{tabular}

La rigidez institucional dificulta la adaptación a las necesidades concretas de los jóvenes: «yo quería hacer amistades fuera del centro, pero las normas no me permitían ni usar teléfono móvil dentro, menos aún salir sola», manifiesta una participante ( $\left.\mathrm{RV}_{3} \mathrm{E}_{2}\right)$. Los seguimientos longitudinales muestran las dificultades que, a causa de la normativa 
de los recursos, tiene la juventud tutelada para mantener relaciones normalizadas con población externa al recurso de protección: «la normativa impide que puedan hacer cosas como otros chicos de su edad. He llevado casos en los que no pueden ni salir al cine ellas solas. Tengo que prohibírselo, siendo consciente de lo negativo que es», dice una profesional (GD1P1). «Yo tengo novia de aquí, pero al principio fue difícil, sobre todo cuando estaba en el centro: casi nunca podía verla», comenta un joven $\left(\mathrm{RV}_{4} \mathrm{E} 1\right)$.

En España, el Sistema de Protección a la Infancia y a la Adolescencia tiene pendiente realizar profundas mejoras en la atención que brinda a la juventud no nacional en dificultad social (tabla 5). La permanencia de modelos asistencialistas en los recursos de protección potencia una contradicción en la formas de pedagogía que ponen en práctica los equipos. En este sentido, se observa que los jóvenes que transitan por recursos actúan de forma contradictoria (SL1, SL2, SL3). «En los centros te dicen cosas muy diferentes, según el centro del que se trate. Hasta que creces no te das cuenta de quién tenía razón», comenta un joven $\left(\mathrm{RV}_{1} \mathrm{E}_{1}\right)$. «Varias veces he insistido en la conveniencia de trabajar la emancipación, pero trabajamos desde la sobreprotección. Algunas compañeras comparten lo que digo, pero manda quien manda», dice una educadora $\left(\mathrm{GD}_{2} \mathrm{P}_{5} \mathrm{P}\right)$. Sería conveniente que la transición a la vida adulta fuera un objetivo prioritario para todos los equipos del Sistema de Protección. La situación en la que se emancipa la juventud en dificultad es la muestra de la eficiencia de su actuación.

Los resultados apuntan a la conveniencia de optar por planificaciones de acompañamiento que tengan mayor duración. Los seguimientos nos aproximan a proyectos educativos cortoplacistas. El itinerario de la persona acompañada es contemplado durante el tiempo previsto de intervención, pero hay carencia de ambición en metas destacables para el futuro personal (SL1, Sl2, SL3). «Nos limitamos a trabajar lo inmediato, sin pensar en lo que vendrá», manifiesta una profesional (GD2P1P). Esta situación tiene consecuencias en la transición a la vida adulta. «En los centros están mucho más preocupadas por los estudios que por cómo aprendemos a vivir solos», dice una joven ( $\left.\mathrm{RV}_{3} \mathrm{E}_{3}\right)$. Otra participante afirma que «hasta que entras en la vivienda [recurso especializado en la emancipación], nadie te habla de prepararte para la vida: vives como aislada de la vida real» $\left(\mathrm{RV}_{2} \mathrm{E}_{1}\right)$. 


\section{Tabla 5}

Aspectos pendientes de mejora en la atención a adolescentes y jóvenes en dificultad social

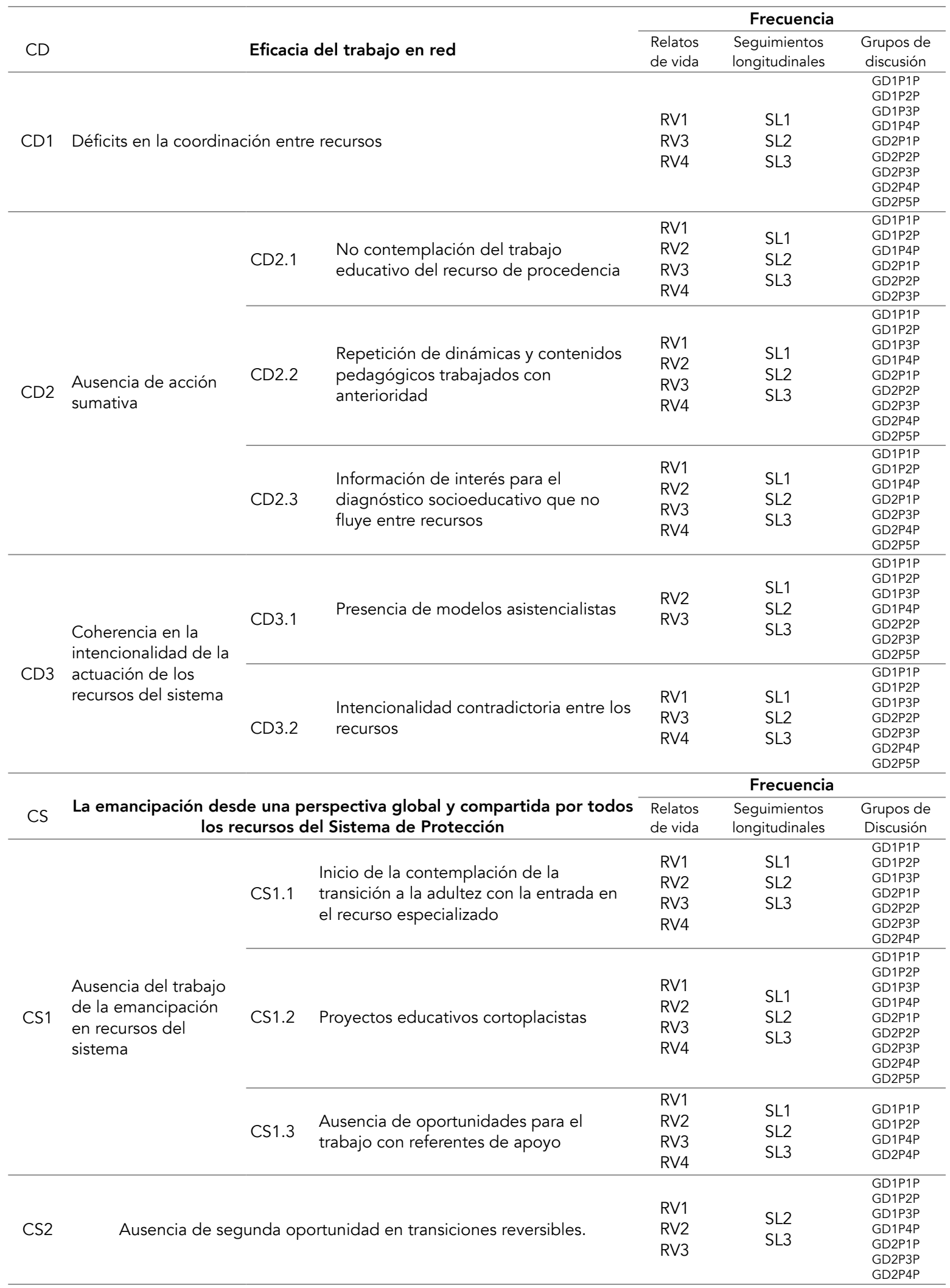


El trabajo de la emancipación está exclusivamente centrado en el hacer socioeducativo del programa especializado, y tiene un escaso papel en la intencionalidad pedagógica de los recursos previos. Las propias figuras profesionales consideran deficitaria la preparación para la vida adulta: «llegan a los 17 años sin tener previsto prácticamente nada, y luego, en el programa hay poco tiempo para trabajar tantas cosas», comenta una profesional $\left(\mathrm{GD}_{2} \mathrm{P}_{1} \mathrm{P}\right)$. Las transiciones a la adultez analizadas coinciden con los denominados procesos de emancipación precarios (Casal, García, Merino, \& Quesada, 2006), mientras los recursos de protección, excepto el especializado en emancipación, permanecen ajenos a esta cuestión. Durante la investigación se recogieron manifestaciones que describen intervenciones negativas para el proyecto de emancipación: «hay centros en los que la sobreprotección es evidente: están como en una burbuja, y al salir a la realidad vienen los problemas», dice una educadora $\left(\mathrm{GD}_{1} \mathrm{P}_{1} \mathrm{P}\right)$.

El Sistema de Protección tiene pendiente consolidar la segunda oportunidad, aspecto imprescindible en el marco de una política institucional que apueste por la eficiencia de los recursos públicos, así como por la garantía de los derechos fundamentales de la adolescencia migrante. Trabaja con una perspectiva lineal y unidireccional. La intervención se organiza como secuencias temporales que entran en contradicción con la situación real. «Vas trabajando con ellos los diferentes aspectos para vivir solos, pero a veces deciden irse, y ahí acaba el trabajo», comenta una profesional $\left(\mathrm{CD}_{1} \mathrm{P}_{3} \mathrm{P}\right)$. Otra educadora completa el argumento manifestando que «la mayoría de las veces, cuando salen así, en unas semanas quieren regresar al sistema, pero la burocracia ya no les deja» (GD1P1P). Este hecho rompe uno de los principios básicos del acompañamiento socioeducativo: el aprovechamiento de la oportunidad. Las necesidades sociales y la organización administrativa entran en conflicto. En una acción cuya intencionalidad es el empoderamiento individual y colectivo, la mejora de las fortalezas (Gutiérrez-Sánchez \& Diz-Casal, 2017) es determinante. La juventud que vive en solitario un corto periodo de tiempo fuera del sistema valora en mayor medida las oportunidades que desde los recursos se le facilitan: «cuando están fuera una temporada, están en el punto de aprovechar las oportunidades», manifiesta una profesional $\left(\mathrm{G}_{2} \mathrm{P}_{2} \mathrm{P}\right)$.

\section{Discusión}

Los resultados apuntan a que la individualización debe ser el centro de la acción socioeducativa, lo que coincide con investigaciones previas (Melendro et al., 2013). El pro- 
tagonismo del trabajo educativo individual se acentúa por el carácter particular de cada trayectoria migratoria (Fuentes, 2014), lo cual condiciona la planificación de las metas. Garantizar el derecho a una participación activa favorece que las cuestiones individuales de cada joven cobren protagonismo durante la intervención. Contar con la voz de la juventud durante el acompañamiento garantiza el respeto al proyecto de vida (Jiménez \& Dios-Izquierdo, 2013) y esto hace de las metas el elemento motivacional que dinamiza la apuesta estratégica de la acción socioeducativa. Los resultados coinciden con otros estudios en que la acción institucional no siempre respeta prioridades del proyecto migratorio, tales como la inserción laboral (Bravo \& Santos-González, 2017). Así que las administraciones implicadas tienen pendiente garantizar que la participación de la juventud migrante sea efectiva.

Confianza y relación con los equipos educativos son dimensiones que condicionan la calidad del acompañamiento. Los resultados apuntan a que la estabilidad de los equipos educativos es determinante en la configuración de un marco de seguridad personal que favorezca la consecución de los objetivos educativos. Las figuras profesionales son un factor de educabilidad (Carbonero et al., 2011) y se configuran como adultos de referencia para los jóvenes en protección con déficits de referentes en sus contextos naturales. La confianza también proporciona garantías de veracidad de la información facilitada por la figuras profesionales a los menores extranjeros (Bravo \& Santos-González, 2017). Los resultados señalan que ese clima de seguridad y relación de apoyo facilita una mejor comprensión de las demandas de la sociedad de acogida.

Coincidimos con investigaciones previas en que continúa pendiente una acción con mayor peso en las fortalezas (De la Paz, 2011; Manzani \& Arnoso, 2014), la cual se apoye en las estrategias desarrolladas por el joven durante el proceso migratorio (Senovilla, 2014). Los resultados destacan la conveniencia de que las diferencias del proceso vital de cada migrante sean tenidas en cuenta como aspectos que enriquecen el acompañamiento socioeducativo. Constatamos que los elementos discrepantes producen un conflicto entre los jóvenes y el Sistema de Protección, que se traduce en incidencias negativas para las pretensiones del participante. Las figuras profesionales apuestan por visualizar el choque cultural como una oportunidad de aprendizaje compartido. Los hallazgos coinciden con Silva et al. (2018) en destacar la importancia de que la juventud en dificultad social sienta que su aporte a la sociedad en la que reside es reconocido.

En el trabajo se constatan déficits en la coordinación entre los recursos de protección, los cuales actúan como unidades independientes. Se prioriza la derivación (Melen- 
dro et al., 2017) en lugar del trabajo coordinado. En todos los relatos de vida y seguimientos longitudinales se constataron repeticiones de actuaciones en diferentes recursos del Sistema, así como desconocimiento del trabajo realizado por los equipos previos. La ausencia de coordinación es incluso mayor en el caso de actores externos al Sistema de Protección, como es el caso de la escuela. Los resultados demuestran que el sistema educativo gallego permanece ajeno a la dimensión social. Los condicionantes específicos de la juventud migrante latinoamericana no están presentes en la actuación de los equipos docentes, lo que perjudica la inserción escolar del alumnado con necesidades socioeducativas; por ende, está pendiente una respuesta a las demandas sociales del alumnado (Díaz-Alzate \& Mejía-Zapata, 2018). Sería positivo que la escuela atendiese el factor social, ya que si se cubriera esta dimensión se favorecería la inserción escolar (Serrate et al., 2017).

Se comprueban retrasos en el acompañamiento socioeducativo, producto de los trámites administrativos necesarios para las autorizaciones de residencia y de trabajo que se contemplan en la legislación específica en materia de extranjería. Los protocolos internacionales en vigor destacan la importancia de optimizar los tiempos de intervención (Comité de los Derechos del Niño, Naciones Unidas, 2005). Conviene recordar que no es oportuno retrasar el aprovechamiento de las oportunidades formativas o laborales, ni siquiera en caso de que continúen pendientes los diferentes procesos de valoración y clarificación de la situación legal (Ministerio de Sanidad, Servicios Sociales e Igualdad, 2016).

Investigaciones previas concluyen que, en su mayoría, la juventud migrante en protección no ha contado con apoyo en el proceso de salida del sistema (Bravo \& SantosGonzález, 2017). El análisis de las estadísticas gallegas nos permite constatar un incremento del número de jóvenes de origen latinoamericano que transita a la vida adulta con el apoyo del programa específico de emancipación (Igaxes3, 2016). El trabajo apunta a una mejora de esta cuestión en Galicia, si bien sería conveniente continuar incrementando el número de plazas disponibles en recursos especializados en la transición a la vida adulta.

En trabajos de otros autores se ha analizado la situación asociativa de la comunidad colombiana en España, así como el creciente papel de estas organizaciones en el contexto de acogida (Lacomba \& Cloquell, 2017). Sin embargo, las oportunidades de acción de las redes de asociaciones colombianas parecen no tener incidencia directa en la intervención administrativa con adolescentes compatriotas en situación de vulnerabilidad social. Lo expuesto supone una dificultad añadida al acompañamiento que se presta a la adolescencia migrante. En los seguimientos longitudinales se observó la intención de los 
equipos educativos de buscar redes de apoyo. En todos los casos, los esfuerzos profesionales fueron infructuosos. Las políticas públicas tienen pendiente la promoción del tejido asociativo de las comunidades latinoamericanas en España, para que, entre otras cuestiones, puedan implicarse en la facilitación de apoyos a la juventud y adolescencia en vulnerabilidad social.

\section{Conclusiones}

El Sistema de Protección tiene pendiente dar una respuesta efectiva a las necesidades de la juventud de origen latinoamericano en dificultad social. La presente investigación nos aproxima a la realidad diferenciada que vive la juventud en riesgo de exclusión nacida en España y la procedente de América Latina. Las propuestas de actuación administrativa para ambos colectivos coinciden, si bien no contemplan las demandas específicas de cada caso. Comprobamos una discrepancia entre la rigidez burocrática, que actúa implacable, sin atender las necesidades individuales de la adolescencia latinoamericana y los esfuerzos de los equipos educativos que pretenden apoyar los procesos de integración social. La rigidez del Sistema de Protección limita los procesos de empoderamiento de los y las jóvenes, dificultando todavía más el logro de sus expectativas, a pesar del esfuerzo de los educadores y educadoras.

La aplicación del marco legislativo sobre extranjería no facilita la integración social y, al mismo tiempo, reduce las oportunidades de la juventud no nacional. Los equipos educativos se ven obligados a desarrollar estrategias que minimicen las frustraciones de la juventud latinoamericana derivadas de las exigencias burocráticas. El marco legal entra en conflicto con las motivaciones y previsiones con las que se planificó el proyecto migratorio. Los recursos de protección carecen de proyectos específicos para dar respuesta a esta juventud. Las figuras profesionales centran sus esfuerzos en superar las dificultades derivadas de la propia estructura y organización del Sistema de Protección, facilitando un acompañamiento individualizado que se adapte a las demandas de la juventud. El Estado tiene pendiente deshacer estas barreras burocráticas que dificultan el trabajo de las figuras profesionales y, para la juventud migrante, extenuante la superación de las metas de los itinerarios.

Se estima que la construcción de un clima de confianza y seguridad para los adolescentes es un factor clave. Las consecuencias de las vivencias propias de la situación de exclusión, agravadas por el desconocimiento y la desconfianza hacia una realidad social 
ajena - e incluso en ocasiones valorada como hostil - exigen la configuración de espacios de confort y seguridad personal. La autopercepción de protección facilita asumir los proyectos educativos necesarios para superar las metas personales. Realismo y adaptabilidad a las complejas condiciones contextuales requieren del desarrollo de procesos relacionales que presenten mayor carga positiva y reduzcan el peso del conflicto, producto de la frecuente imposición normativa que no comprende la utilidad educativa de la norma. Se trata de apostar por las fortalezas personales para impulsar los caminos de la emancipación. Las figuras profesionales valoran que la juventud reconozca la utilidad de la acción protectora; esto es determinante para que los profesionales se sientan motivados y comprometidos con el proyecto educativo. El carácter pragmático de la juventud afectada por la exclusión social precisa una respuesta socioeducativa que facilite oportunidades valoradas como útiles para el proyecto migratorio. La horizontalidad en la relación con los equipos educativos y la participación real son dos elementos que favorecen el respeto a las pretensiones de la persona acompañada que, recordemos, es la última responsable de la consecución de las metas del proyecto educativo.

La falta de adecuación de los procesos burocráticos a la realidad de los itinerarios de emancipación se evidencia en transiciones a la adultez reversibles, para las que el Sistema de Protección no tiene respuestas adecuadas. La juventud de origen latinoamericano, especialmente durante la emancipación, carece de figuras referenciales de apoyo. La escasa presencia de tejido asociativo latinoamericano, así como de emigrantes adultos que participen como voluntarios en programas de referentes, condiciona la estrategia socioeducativa de los equipos profesionales. La actuación de los recursos no cuenta con el apoyo de referentes externos. Esta situación es diferente a la de otra juventud migrante. En el caso de la juventud del África subsahariana, la implicación de los compatriotas adultos posibilita el acceso a más oportunidades de los participantes tutelados, especialmente en lo relativo a referenciación social y mentoría. Sería positivo que la administración pública potenciase políticas que favorezcan la dinamización de asociaciones de emigrantes latinoamericanos, las cuales podrían contribuir a la inclusión social de la juventud en exclusión.

El Sistema de Protección a la Infancia y a la Adolescencia de España tiene pendiente desarrollar mecanismos específicos que respondan a las necesidades de la juventud migrante de América Latina. Resulta especialmente destacable la ausencia de lo que venimos denominando segunda oportunidad. Se trata de facilitar apoyos a la juventud ya emancipada, cuya situación de exclusión social es manifiesta. Estos jóvenes no cuentan con redes sociales naturales que les faciliten soporte y necesitan acompañamiento socio- 
educativo para lograr las metas de su proyecto migratorio. En definitiva, se trataría de hacer más eficaz el Sistema de Protección para posibilitar apoyos en el momento en que la juventud es más receptiva a la intervención de este. La valoración de las oportunidades vitales facilitadas por los equipos educativos es mayor cuando se permanece un tiempo fuera del Sistema de Protección. La actuación protectora pretende facilitar la superación de la situación de vulnerabilidad. Es oportuno que la administración configure estrategias que favorezcan el cumplimiento de dicho objetivo. La finalidad es posibilitar, con realismo, actuaciones de los recursos de protección que favorezcan la superación de las metas de cada itinerario migratorio. El empoderamiento de la juventud migrante de América Latina, que en su día fue tutelada, posibilitará su implicación en acciones de transformación social; lo que, a su vez, podrían apoyar a otros adolescentes que posteriormente se encuentren tutelados por la entidad pública de España.

En cuanto a las limitaciones del presente estudio, este se realizó en Galicia y fue apoyado con recursos del Departamento de Análisis e Intervención Psicosocioeducativa de la Universidad de Vigo. Teniendo en cuenta las características específicas del Sistema de Protección español, los resultados son propios del territorio gallego, así que podría haber diferencias en las actuaciones protectoras de otras comunidades autónomas.

\section{Referencias}

Báez, J. (2007). Investigación cualitativa. Esic.

Bravo, A., \& Santos-González, I. (2017). Menores extranjeros no acompañados en España: necesidades y modelos de intervención. Psychosocial Intervention, 26(1), 55-62. https:// doi.org/10.1016/j.psi.2015.12.001

Carbonero, M., Martín-Antón, M., \& Reoyo, N. (2011). El profesor estratégico como favorecedor del clima de aula. European Journal of Education and Psychology, 4(2), 133-142. https://doi.org/10.30552/ejep.v4i2.69

Casal, J., García, M., Merino, M., \& Quesada, M. (2006). Aportaciones teóricas y metodológicas a la sociología de la juventud desde la perspectiva de la transición. Papers, Revista de Sociología, 79, 21-48. https://doi.org/10.5565/rev/papers/v79no.798

Comité de los Derechos del Niño, Naciones Unidas. (2005). Observación general n. 6 CRC/ $G C / 2005 / 6$, del 1.ํㅡ de septiembre de 2005, relativa al trato de menores no acompañados y separados de su familia fuera de su país. Naciones Unidas. 
De la Paz, P. (2011). La intervención en trabajo social desde la perspectiva de las fortalezas. Cuadernos de Trabajo Social, 24, 155-163. https://doi.org/10.5209/rev_cuts.2011.v24.36865

Denzin, N., \& Lincoln, Y. (2008). Strategies of qualitative inquiry. Sage.

Díaz-Alzate, M. V., \& Mejía-Zapata, S. I. (2018). La mirada de los adolescentes al modelo de habilidades para la vida. Revista Latinoamericana de Ciencias Sociales, Niñez y Juventud, 16(2), 709-718. https://doi.org/10.1160o/1692715x.16205

Fuentes, R. (2014). Menores extranjeros no acompañados (Mena). Revista Internacional de Trabajo Social y Bienestar, 3, 105-111.

Gimeno, C. (2018). Retos de la acogida residencial a menores que migran solos: hacia un trabajo social transnacional. Cuadernos de Trabajo Social, 31(1), 95-108. https://doi.org/ $10.5209 /$ cuts. 56005

Gutiérrez-Sánchez, J. D., \& Diz-Casal, J. (2017). Cooperación internacional en Marruecos: empoderamiento para insertar sociolaboralmente a jóvenes en contextos de riesgo. Cuadernos de Trabajo Social, 30(1), 163-174. https://doi.org/10.5209/cuts.50352

Holland, J., Thomson, R., \& Henderson, S. (2006). Qualitative longitudinal research: A discussion paper. London South Bank University.

Hopkins, P., \& Hill, M. (2010). The needs and strengths of unaccompanied asylumseeking children and young people in Scotland. Child \& Family Social Work, ${ }_{15}(4)$, 399-408. https://doi.org/10.1111/j.1365-2206.2010.00687.x

Igaxes3. (2016). Memoria anual del programa Mentor. Igaxes3.

Jiménez, L., \& Dios-Izquierdo, J. (2013). Lo que se oculta detrás de la categoría menores marroquíes no acompañados: miedos cruzados, contradicciones europeas y consecuencias para el trabajo social. Cuadernos de Trabajo Social, 26(1), 193-202. https:// doi.org/10.5209/rev_cuts.2013.v26.n1.41667

Lacomba, J., \& Cloquell, A. (2017). Asociaciones de inmigrantes, Estados y desarrollo entre España y Colombia: ¿un nuevo campo social transnacional? Revista de Estudios Sociales, 61, 44-57. https://doi.org/10.7440/res61.2017.04

López, G. (2018). La construcción de la identidad: menores extranjeros no acompañados, en contextos de protección. RES, Revista de Educación Social, 27, 71-92. https://doi.org/ $10.21696 / \mathrm{rcs} 18152018758$

Manzani, L., \& Arnoso, M. (2014). Bienestar psicosocial en menores y jóvenes extranjeros sin referente familiar adulto: factores de riesgo y de protección. Norte de Salud Mental, $12,33-45$.

Martín, J. M., Alonso, I., \& Tresserras, A. (2016). Aportaciones del paradigma de resiliencia a la acción socioeducativa: el caso del Centro de Menores Extranjeros no Acompa- 
ñados Zabaloetxe. Pedagogía Social, Revista Interuniversitaria, 28, 157-168. https:// doi.org/10.7179/psri_2016.28.12

Martínez, M. (2006). Validez y confiabilidad de la metodología cualitativa. Paradigma, $27(2), 7-34$.

Melendro, M., De Juanas, A., \& Rodríguez, A. E. (2017). Déficits de la intervención socioeducativa con familias de adolescentes en riesgo de exclusión. Bordón. Revista de Pedagogía, 69(1), 123-138. https://doi.org/10.13042/Bordon.2016.48596

Melendro, M., González, A. L., \& Rodríguez, A. E. (2013). Estrategias eficaces de intervención socioeducativa con adolescentes en riesgo social. Pedagogía Social, 22, 105-121. https://doi.org/10.7179/psri_2013.22.08

Miles, M., \& Huberman, A. (2007). Analyse des donnes qualitatives. De Boeck.

Ministerio de Sanidad, Consumo y Bienestar Social. (2018). Boletín de datos estadísticos de medidas de protección a la infancia. http://www.observatoriodelainfancia.mscbs.gob.es/ productos/pdf/Boletin_2O_DEFINITIVO.pdf

Ministerio de Sanidad, Servicios Sociales e Igualdad. (2016). Vy VI Informe de Aplicación de la Convención sobre los Derechos del Niño de NN.UU. y sus protocolos facultativos. https:// www.observatoriodelainfancia.es/oia/esp/documentos_ficha.aspx?id=4961

Monteros, S. (2014). La gubernamentabilidad de la infancia extranjera. En A. Jiménez, J. J. Leiva, E. Moreno, \& A, Pantoja (coords.), Infancia en contextos de riesgo (pp. 71-77). GEU.

Puig, G., \& Rubio, J. L. (2015). Tutores de resiliencia: dame un punto de apoyo y moveré mi mundo. Gedisa.

Puigvert, L. (2014). Preventive socialization of gender violence: Moving forward using the communicative methodology of research. Qualitative Inquiry, 20(7), 839-843. https://doi.org/10.1177/1077800414537221

Ruiz, A. C., Palma, M. O., \& Vives, L. C. (2019). Jóvenes inmigrantes extutelados: el tránsito a la vida adulta de los menores extranjeros no acompañados en el caso español. Ehquidad. International Welfare Policies and Social Work Journal, 12, 31-52. https://doi.org/10.15257/ehquidad.2019.0009

Ruiz, J. I. (2012). Metodología de la investigación cualitativa. Deusto.

Sandin, M. P. (2003). Investigación cualitativa en educación: fundamentos y tradiciones. McGraw-Hill.

San Martín, D. (2014). Teoría fundamentada y Atlas.ti: recursos metodológicos para la investigación educativa. Revista Electrónica de Investigación Educativa, 16(1). 104-122. http://redie.uabc.mx/voli6no1/contenido-sanmartin.html 
Schütz, F., Castellá, J., Bedin, J., \& Montserrat, C. (2015). Subjective well-being of children in residential care centers: Comparison between children in institutional care and children living with their families. Psicoperspectivas, Individuo y Sociedad, 14(1), 19-3o. https://doi.org/10.5027/psicoperspectivas-Voli4-Issue1-fulltext-517

Senovilla, D. (2014). Menores no acompañados y no protegidos: resultados de una investigación en cuatro estados europeos. REMHU: Revista Interdisciplinar da Mobilidade Humana, 22(42), 81-96. https://doi.org/10.1590/s1980-85852014000100006

Serrate, S., González, M., \& Olmos, S. (2017). La acción socioeducativa interdisciplinar en la etapa de educación secundaria: situación y necesidades profesionales. Revista de Educación, 376, 200-228. https://doi.org/10.4438/1988x-re-2017-376-342

Silva, L., Aristizábal, A., Gómez, M., González, Y., Acevedo, C., Ortiz, Y., Rodríguez, N., \& Campo, N. (2018). Reconstrucción de experiencias y percepciones propias de jóvenes habitantes de calle. Revista Latinoamericana de Ciencias Sociales, Niñez y Juventud, 16(2), 8o9-823. https://doi.org/10.1160o/1692715x.16211

Steinar, K. (2011). Las entrevistas en investigación cualitativa. Morata.

Strauss, A., \& Corbin, J. (2002). Bases de la investigación cualitativa: técnicas y procedimientos para desarrollar la teoría fundamentada. Universidad de Antioquia.

Tójar, J. C. (2006). Investigación cualitativa: comprender y actuar. La Muralla.

Xunta de Galicia. (2016). Estadísticas de protección de menores. www.xunta.gal 